\title{
Highlights from the latest articles in nanomaterial-based therapies for targeting cancer stem cells
}

\section{Selectively targeting stem cells using graphene oxide \\ Evaluation of: Fiorillo M, Verre AF, lliut $\mathrm{M}$ et al. Graphene oxide selectively targets cancer stem cells, across multiple tumor types: implications for non-toxic cancer treatment, via 'differentiation- based nano-therapy'. Oncotarget 6(6), 3553-3562 (2015).}

Cancer stem cells (CSCs) represent a key challenge in cancer treatment. These cells are strongly implicated in tumor recurrence and metastasis, making them obvious targets for improved therapeutic approaches. The low abundance of CSCs and their rapid acquisition of drug resistance make these cells very challenging targets. The unique threat (and opportunity) presented by CSCs arises from their ability to differentiate. This 'stemness' provides an alternative therapeutic strategy: if CSCs can be induced to differentiate, they lose many of the properties that make them so dangerous. The fact that this approach targets a phenotypic change and not cytotoxicity presents the possibility of effective treatment with greatly reduced off-target effects.

In a recent study, Fiorillo et al. examined the use of graphene oxide (GO) as a therapeutic strategy against CSCs. In this study, the researchers used two highly polydisperse GO populations: small $(200 \mu \mathrm{nm}-2 \mu \mathrm{m})$ and large $(5-20 \mu \mathrm{m})$. Stemness was determined using a tumor spheroid assay, where the ability of tumor cells to proliferate in an anchorage-independent fashion provides a phenotypic screen for CSCs. Using a set of five different cell lines, the researchers first determined toxicity of the GOs to the cells, observing no toxicity over the range studied
$(0-100 \mu \mathrm{g} / \mathrm{ml})$. By contrast, dose-dependent decreases in spheroid generation were observed in all cell lines studied. Mechanistic studies indicated that the GOs inhibited signal transduction pathways, including WNT, Notch and STAT that were presumed to result in the differentiation of the CSCs.

This study presents an excellent example of the targeting of CSC phenotype as a therapeutic strategy, where a material that is relatively nontoxic could have profound therapeutic effects. Engineered nanomaterials provide a diverse library for the development of potent and better-characterized therapies against CSCs. Screening of this vast library, however, would be very cumbersome using the spheroid assay, creating the need for better tools for monitoring CSC differentiation.

\section{Targeting cancer stem cells with nanoparticle-delivered microRNAs Evaluation of: Kouri FM, Hurley LA, Daniel WL et al. miR-182 integrates apoptosis, growth and differentiation programs in glioblastoma. Genes Dev. 29(7), 732-745 (2015).}

The 'holy grail' of CSC research is to identify therapeutics that target this population of cells specifically, and to optimize the delivery of these therapeutics into tumors. miRs have considerable potential in this context because they have the ability to silence the expression of genes that control stem cell properties. This approach, however, requires the identification of specific miRs that target CSCs and the development of methods to deliver these miRs effectively into tumors. This latter requirement has hindered the use of miRs for cancer therapy and it is particularly challenging for brain cancers because therapeu-
Tatsuyuki Yoshii $i^{\ddagger 1}$, Yingying Geng $^{\ddagger, 1}$, Ngoc DB Le', Hira Lal Goel $^{2}$, Arthur M Mercurio*,2 \& Vincent M Rotello**,1 'Department of Chemistry, University of Massachusetts Amherst, 710 North Pleasant Street, Amherst, MA 01003, USA

2Department of Molecular, Cell \& Cancer Biology, University of Massachusetts Medical School, Worcester, MA 01605, USA

*Author for correspondence: Arthur.Mercurio@umassmed.edu **Author for correspondence: rotello@chem.umass.edu

${ }^{\ddagger}$ Authors contributed equally 
tics must be able to cross the blood-brain (BB) and blood-tumor (BT) barriers.

Recently, Khouri et al. demonstrated that miR-182 regulates the apoptosis, growth and differentiation of glioblastoma cells. A particularly interesting observation from this study was that miR-182 inhibits 'stemness' in vitro and reduces the expression of key stem cell markers. Subsequently, they exploited the use of a spherical nucleic acids (SNAs) nanotechnology platform that they had developed. SNA gold nanoparticles can cross the BB and BT barriers and deliver siRNAs into tumors in the absence of significant toxicity or immunogenicity. In the current study, they were able to modify the SNA nanotechnology to generate gold nanoparticles covalently functionalized with mature miR-182 duplexes. Using glioblastoma xenografts, they demonstrated that the miR-182-SNAs were able to cross the $\mathrm{BB}$ and $\mathrm{BT}$ barriers and disseminate throughout the glioma parenchyma. As a result, tumor burden was reduced significantly.

This study highlights the usefulness of nanotechnology to deliver therapeutics efficiently and effectively into tumors, especially the ability to penetrate the formidable BB and BT barriers. Although miR-182 may not target CSCs specifically, it clearly affects stemness. This study should stimulate work on other miRs that have the potential to target CSCs and to use nanotechnology approaches for their delivery into tumors.

\section{pH-sensitive, chitosan-based nanoparticles provide specificity in targeting cancer stem cells}

Evaluation of: Rao W, Wang $\mathrm{H}$, Han J et al. Chitosan-decorated doxorubicin-encapsulated nanoparticle targets and eliminates tumor reinitiating cancer stem-like cells. ACS Nano 9(6), 5725-5740 (2015).

CSCs are difficult to target because of their multidrug resistance. CSCs overexpress transmembrane ATPbinding cassette transporters that actively pump free anticancer drugs out of cells [1]. In addition to drug efflux, some intrinsic properties of CSCs such as dormancy and resistance to apoptosis also make CSCs hard to tackle $[2,3]$. Despite the challenges, much effort is being made to selectively target CSCs.

In a recent publication, Rao et al. developed a $\mathrm{pH}$ sensitive polymeric nanoparticle decorated with chitosan as a vehicle to specifically deliver doxorubicin, an anticancer drug, to breast CSCs. Many CSCs express high levels of CD44 receptor, which functions as a receptor for hyaluronic acid (HA). Based on this knowledge, they decorated the surface of nanoparticles with HAfunctionalized chitosan and tested these nanoparticles on mammospheres derived from MCF-7 human breast cancer cells. Mammospheres are enriched in CSCs. Immunostaining results showed binding between nanoparticle-encapsulated doxorubicin (nDox) and CD44 receptors via HA-functionalized chitosan. Once inside the cells, the weakly acidic $\mathrm{pH}$ environment stimulated nanoparticles to release doxorubicin, and the accumulated doxorubicin effectively reduced the $\mathrm{CD} 44^{+}$subpopulation of cells. However, targeting CD 44 on CSCs could be a concern because CD 44 can also be found on normal cells, potentially leading to many adverse effects. Interestingly, nDox did not show any binding to noncancerous stem cells. This result might be attributed to the possibility that normal and cancer stem cells express different splice variants of CD44. Subsequently, the authors applied their system in vivo using orthotopic tumor-bearing mice and showed that $\mathrm{nDox}$ has an enhanced antitumor efficacy with low systemic toxicity.

The chitosan-decorated nanoparticles used in this publication could be a promising approach for targeting CSCs, which are likely responsible for tumor recurrence. This approach could be used in combination with conventional chemotherapy that targets the bulk population of tumor cells and reduces tumor burden. Going forward, this approach could be improved by identifying markers whose expression is restricted to CSCs and with the use of drugs that target the specific properties of CSCs.

\section{Nanomedicine-based starvation tactics for elimination of cancer stem cells}

Evaluation of: Xu CF, Liu Y, Shen S, Zhu

$\mathrm{YH}$, Wang J. Targeting glucose uptake with siRNA-based nanomedicine for cancer therapy. Biomaterials 51, 1-11 (2015).

A tumor microenvironment that is low in glucose increases the frequency of CSCs and cells with stem cell properties are able to survive in this adverse condition much better than non-CSCs. This phenomenon has been observed in gliomas and other solid tumors. The glucose transporter (GLUT3) is highly expressed in glioma stem cells [4], which enables these cells to increase their uptake of glucose and survive in a low glucose microenvironment. Although GLUT-3 is an attractive therapeutic target for this reason, no synthetic inhibitors of this transporter have been developed and alternative approaches are required.

In a recent report, $\mathrm{Xu}$ et al. found that nanoparticles carrying siRNA $\left(\mathrm{NP}_{\text {siRNA }}\right)$ targeting GLUT3 can eliminate glioma cells, as well as the bulk population of tumor cells. They formulated $\mathrm{NP}_{\text {siRNA }}$ by double emulsification with cationic amphiphile and poly(ethylene glycol)-b-poly(D,L-lactide) (PEG-PLA) to form stable approximately $125 \mathrm{~nm}$ particles. Using this cationic 
lipid-assisted PEG-PLA NP system, the siRNA was efficiently delivered into glioma cell lines U87MG and U251. This approach decreased the expression of GLUT3 with a concomitant reduction of glucose uptake and significant metabolic inhibition. Consequently, cell viability of both cancer stem cells and cancer cells in each cell line decreased, with a higher cell death rate in CSCs as shown by CSC biomarker $\mathrm{CD} 133^{+}$. When this system was applied to mice bearing U87MG xenografts, tumor volume was significantly reduced. Gene expression analysis revealed that nanoparticle delivery of the GLUT3 siRNA reduced the expression of stem cell markers in vitro and in vivo.

This study provides a new strategy for CSC treatment using nanoparticle-delivery of siRNA to target metabolism. Further screening of siRNA carriers

\section{References}

1 Chuthapisith S, Eremin J, El-Sheemey M, Eremin O. Breast cancer chemoresistance: emerging importance of cancer stem cells. Surg. Oncol. 19(1), 27-32 (2009).

2 Naumov GN, Bender E, Zurakowski D et al. A model of human tumor dormancy: an angiogenic switch from the nonangiogenic phenotype. J. Natl Cancer Inst. 98(5), 316-325 (2006). such as other polymers and nanoparticles could lead to a more effective delivery to target CSCs. Moreover, this strategy may be useful for other types of cancers that exhibit enhanced GLUT3 expression. Importantly, this study indicates that the downregulation of GLUT3 can be achieved with minimal toxicity.

\section{Financial \& competing interests disclosure}

The authors have no relevant affiliations or financial involvement with any organization or entity with a financial interest in or financial conflict with the subject matter or materials discussed in the manuscript. This includes employment, consultancies, honoraria, stock ownership or options, expert testimony, grants or patents received or pending, or royalties.

No writing assistance was utilized in the production of this manuscript

3 Bapat SA. Human ovarian cancer stem cells. Reproduction 140(1), 33-41 (2010).

4 Flavahan WA, Hitomi M, Rahim N et al. Brain tumor initiating cells adapt to restricted nutrition through preferential glucose uptake. Nat. Neurosci. 16(10), 1373-1382 (2013). 